\title{
The Quantum Adiabatic Approximation and the Geometric Phase
}

\author{
Ali Mostafazadeh* \\ Theoretical Physics Institute, University of Alberta, \\ Edmonton, Alberta, Canada T6G 2J1. \\ June 1996
}

\begin{abstract}
A precise definition of an adiabaticity parameter $\nu$ of a time-dependent Hamiltonian is proposed. A variation of the time-dependent perturbation theory is presented which yields a series expansion of the evolution operator $U(\tau)=\sum_{\ell} U^{(\ell)}(\tau)$ with $U^{(\ell)}(\tau)$ being at least of the order $\nu^{\ell}$. In particular $U^{(0)}(\tau)$ corresponds to the adiabatic approximation and yields Berry's adiabatic phase. It is shown that this series expansion has nothing to do with the $1 / \tau$-expansion of $U(\tau)$. It is also shown that the nonadiabatic part of the evolution operator is generated by a transformed Hamiltonian which is off-diagonal in the eigenbasis of the initial Hamiltonian. Some related issues concerning the geometric phase are also discussed.
\end{abstract}

*E-mail: alimos@phys.ualberta.ca 


\section{Introduction}

Since the introduction of the adiabatic geometrical phase by Berry [1], the study of the cyclic evolution of quantum states of non-conservative quantum systems (explicitly time-dependent Hamiltonians) has attracted much attention. By definition a pure cyclic state $|\mathcal{N} ; \tau\rangle\langle\mathcal{N} ; \tau|$ is an eigenstate of the evolution operator $U(\tau)$, i.e.,

$$
\left.U(\tau)=:\left.\sum_{\mathcal{N}} e^{i \beta_{\mathcal{N}}(\tau)}\right|_{\mathcal{N}} ; \tau\right\rangle\left\langle_{\mathcal{N}} ; \tau\right|, \quad \beta_{\mathcal{N}}(\tau) \in[0,2 \pi)
$$

Therefore the quantity of main importance in the study of cyclic states and the accompanying phases $\beta_{\mathcal{N}}(\tau)$ is the evolution operator $U(\tau)$. This operator is defined by

$$
|\psi(\tau)\rangle=: U(\tau)|\psi(0)\rangle
$$

where $|\psi(\tau)\rangle$ is the solution of the Schrödinger equation. Alternatively, one can define $U(\tau)$ as the solution of

$$
\begin{aligned}
\frac{d}{d \tau} U(\tau) & =-\frac{i}{\hbar} H(\tau) U(\tau) \\
U(0) & =1
\end{aligned}
$$

where $H=H(\tau)$ stands for the Hamiltonian and $\tau \in[0, \infty)$ parameterizes the time. The solution of Eq. (3) can be implicitly expressed in the form:

$$
U(\tau):=\mathcal{T} e^{-\frac{i}{\hbar} \int_{0}^{\tau} H(t) d t}
$$

where $\mathcal{T}$ denotes the time-ordering operator [2].

The purpose of this article is two fold. First, a precise definition of an adiabaticity parameter $\nu$ will be given. This is a parameter which quantifies the rapidity of the timedependence of the Hamiltonian. Next, a series expansion of the evolution operator $U(\tau)$ will be proposed whose $\ell$-th term is at least of the order $\nu^{\ell}$. In particular the adiabatic approximation:

$$
U(\tau) \approx \sum_{n} e^{i \alpha_{n}(\tau)}|n ; \tau\rangle\langle n ; 0|
$$

is recovered as the first term. In Eq. (可), $|n ; t\rangle$ are the instantaneous eigenstate vectors of the Hamiltonian, i.e.,

$$
H(t)|n ; t\rangle=E_{n}(t)|n ; t\rangle
$$


and

$$
\alpha_{n}(t):=\delta_{n}(t)+\gamma_{n}(t), \quad \delta_{n}(t):=-\frac{1}{\hbar} \int_{0}^{t} E_{n}\left(t^{\prime}\right) d t^{\prime}, \quad \gamma_{n}(t):=i \int_{0}^{t}\left\langle n ; t^{\prime}\left|\frac{d}{d t^{\prime}}\right| n ; t^{\prime}\right\rangle d t^{\prime} .
$$

If the Hamiltonian is $T$-periodical, i.e., $H(T+t)=H(t)$, the $\alpha_{n}(T), \delta_{n}(T)$, and $\gamma_{n}(T)$ are called adiabatic total phase angle, adiabatic dynamical phase angle, and adiabatic geometrical phase or Berry phase angle, respectively [1].

The approach pursued in this article clarifies the misconception that the adiabatic approximation of $U(\tau)$ is given by the zero-th order term in its $1 / \tau$-expansion, i.e., it is only valid for $\tau \rightarrow \infty$. It also provides a convenient framework to perturbatively compute the non-adiabatic corrections to Berry's adiabatic phase.

\section{The Adiabaticity Parameter}

Following Berry [1], consider a parameter-dependent Hamiltonian:

$$
H[R]=\sum_{n} E_{n}[R]|n ; R\rangle\langle n ; R|,
$$

where $R=\left(R^{1}, \cdots, R^{d}\right)$ are real parameters viewed as the coordinates of a parameter space $\mathcal{M}, E_{n}[R]$ are the real eigenvalues and $\{|n ; R\rangle\}$ is a complete orthonormal set of eigenvectors of $H[R]$. In this case a time-dependent Hamiltonian $H(t)$ corresponds to a curve $C:[0, \tau] \rightarrow$ $\mathcal{M}$ in $\mathcal{M}$ :

$$
H(t):=H[C(t)]=H\left[R_{C}(t)\right], \quad \text { with } C(t)=:\left(R_{C}^{1}(t), \cdots, R_{C}^{d}(t)\right)=: R_{C}(t) .
$$

Furthermore, suppose that the spectrum of $H[R]$ for all $R \in \mathcal{M}$ is discrete, the energy eigenvalues $E_{n}[R]$ are non-degenerate, and there is no level-crossing, i.e., for every $t \in[0, \tau]$, $E_{n}\left[R_{C}(t)\right]<E_{n+1}\left[R_{C}(t)\right]$.

In order to define a dimensionless adiabaticity parameter, first one defines a characteristic frequency:

$$
\omega_{c}\left(\tau_{1}, \tau_{2}\right):=\operatorname{Sup}\left\{\left|A_{m n}(t)\right|: n \neq m=0,1, \cdots, \quad t \in\left[\tau_{1}, \tau_{2}\right]\right\} ; \quad\left[\tau_{1}, \tau_{2}\right] \subseteq[0, \tau],
$$

with "Sup" abbreviating supremum (least upper bound) and

$$
A_{m n}(t):=\left\langle m ; t\left|\frac{d}{d t}\right| n ; t\right\rangle
$$




$$
=\frac{\left\langle m ; t\left|\left[\frac{d}{d t} H(t)\right]\right| n ; t\right\rangle}{E_{n}(t)-E_{m}(t)}, \quad \text { for } m \neq n .
$$

Then the desired adiabaticity parameter is defined according to

$$
\nu:=\frac{\hbar \omega_{c}(0, \tau)}{\Delta E}
$$

where $\Delta E$ is a convenient energy scale. For example one can take $\Delta E$ to be the first transition energy of the initial Hamiltonian, i.e., $\Delta E:=E_{1}(0)-E_{0}(0)$.

The main motivation for this definition is the fact that according to Eqs. (9), (11), and (12), $\omega_{c}(0, \tau)$ and therefore $\nu$ involve time derivatives of the Hamiltonian $H(t)$. The use of $\omega_{c}(0, \tau)$ in the definition of the adiabaticity parameter will be self-evident once one examines the evolution operator. The role of $\Delta E$ is to provide a convenient energy $(\hbar \times$ frequency) scale.

It must also be emphasized that by definition, $\nu$ is a "global" quantity which characterizes the time-dependence of the Hamiltonian. In particular, it is neither equal nor proportional to $1 / \tau$. Furthermore, note that although $|n ; t\rangle$ are only determined up to arbitrary $R_{C}(t)$ -

dependent phase factors (gauge transformations along $C$, [2]), $\left|A_{m n}(t)\right|$ with $m \neq n$ and therefore $\omega_{c}\left(\tau_{1}, \tau_{2}\right)$ and $\nu$ are independent of the choice of such phases (they are gaugeinvariant quantities). In other words, $\nu$ is well-defined.

\section{Computation of $U(\tau)$}

Consider the definition of the time-ordered product in (荬):

$$
U(\tau):=\lim _{N \rightarrow \infty}\left[1-\frac{i}{\hbar} H\left(t_{N}\right) \epsilon\right] \cdots\left[1-\frac{i}{\hbar} H\left(t_{k}\right) \epsilon\right] \cdots\left[1-\frac{i}{\hbar} H\left(t_{0}\right) \epsilon\right]
$$

where $\epsilon:=\tau / N$ and $t_{k}:=k \epsilon$, for $k=0,1, \cdots N$. Using the orthonormality and completeness of the energy eigenvectors $|n ; t\rangle$, one can compute:

$$
\begin{aligned}
U(\tau)= & \lim _{N \rightarrow \infty} \sum_{n_{0}, \cdots, n_{N}}\left\{\left|n_{N} ; t_{N}\right\rangle\left\langle n_{N} ; t_{N}\left|\left[1-\frac{i}{\hbar} H\left(t_{N}\right) \epsilon\right]\right| n_{N-1} ; t_{N-1}\right\rangle\left\langle n_{N-1} ; t_{N-1}\right|\right. \\
& \cdots \\
& \left|n_{k} ; t_{k}\right\rangle\left\langle n_{k} ; t_{k}\left|\left[1-\frac{i}{\hbar} H\left(t_{k}\right) \epsilon\right]\right| n_{k-1} ; t_{k-1}\right\rangle\left\langle n_{k-1} ; t_{k-1}\right|
\end{aligned}
$$




$$
\begin{aligned}
& \left.\left|n_{1} ; t_{1}\right\rangle\left\langle n_{1} ; t_{1}\left|\left[1-\frac{i}{\hbar} H\left(t_{0}\right) \epsilon\right]\right| n_{0} ; t_{0}\right\rangle\left\langle n_{0} ; t_{0}\right|\right\} \\
= & \lim _{N \rightarrow \infty} \sum_{n_{0}, \cdots, n_{N}}\left(e^{-\frac{i}{\hbar} \sum_{j=0}^{N} E_{n_{j}}\left(t_{j}\right) \epsilon} \prod_{k=1}^{N}\left\langle n_{k} ; t_{k} \mid n_{k-1} ; t_{k-1}\right\rangle\right)\left|n_{N} ; t_{N}\right\rangle\left\langle n_{0} ; t_{0}\right| .
\end{aligned}
$$

Introducing

$$
\begin{aligned}
K_{n_{N} n_{0}}(\tau) & :=\left\langle n_{N} ; \tau|U(\tau)| n_{0} ; 0\right\rangle \\
& =\lim _{N \rightarrow \infty} \sum_{n_{1}, \cdots, n_{N-1}} e^{-\frac{i}{\hbar} \sum_{j=1}^{N-1} E_{n_{j}}\left(t_{j}\right) \epsilon} \prod_{k=1}^{N}\left\langle n_{k} ; t_{k} \mid n_{k-1} ; t_{k-1}\right\rangle
\end{aligned}
$$

one then has:

$$
U(\tau)=\sum_{m n} K_{m n}(\tau)|m ; \tau\rangle\langle n ; 0|
$$

The computation of the terms in the product in Eq. (14), is straightforward:

$$
\begin{aligned}
\left\langle n_{k} ; t_{k} \mid n_{k-1} ; t_{k-1}\right\rangle & =\delta_{n_{k} n_{k-1}}-\left.\epsilon\left\langle n_{k} ; t\left|\frac{d}{d t}\right| n_{k-1} ; t\right\rangle\right|_{t=t_{k-1}}+\mathcal{O}\left(\epsilon^{2}\right) \\
& =e^{-\epsilon A_{n_{k} n_{k-1}}\left(t_{k-1}\right)} \delta_{n_{k} n_{k-1}}+\epsilon \Delta_{n_{k} n_{k-1}}\left(t_{k-1}\right)+\mathcal{O}\left(\epsilon^{2}\right) \\
& =e^{-\epsilon A_{n_{k} n_{k}}\left(t_{k}\right)}\left[\delta_{n_{k} n_{k-1}}+\epsilon e^{\epsilon A_{n_{k} n_{k}}\left(t_{k}\right)} \Delta_{n_{k} n_{k-1}}\left(t_{k}\right)\right]+\mathcal{O}\left(\epsilon^{2}\right)
\end{aligned}
$$

where

$$
\Delta_{m n}(t):=\left(\delta_{m n}-1\right) A_{m n}(t) .
$$

Substituting (16) in (14), one obtains $2^{N}$ terms which can be arranged in the order of the appearance of different powers of $\epsilon$. In this way one finds only $N$ terms of order $\epsilon^{0}=1$.' These will be denoted by $K_{m n}^{(\ell)}(\tau)$ :

$$
K_{m n}(\tau)=\lim _{N \rightarrow \infty} \sum_{\ell=0}^{N} K_{m n}^{(\ell)}(\tau) .
$$

Performing the algebra one finds:

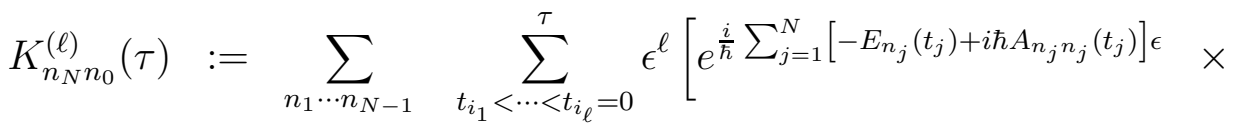

$$
\begin{aligned}
& \delta_{n_{0} n_{1}} \cdots \delta_{n_{i_{1}-2} n_{i_{1}-1}} e^{\epsilon A_{n_{i_{1}} n_{i_{1}}}\left(t_{i_{1}}\right)} \Delta_{n_{i_{1}} n_{i_{1}-1}}\left(t_{i_{1}}\right) \delta_{n_{i_{1}} n_{i_{1}+1}} \cdots \\
& \left.\delta_{n_{i_{\ell}-2} n_{i_{\ell}-1}} e^{\epsilon A_{n_{i_{\ell}} n_{i_{\ell}}}\left(t_{i_{\ell}}\right)} \Delta_{n_{i_{\ell}} n_{i_{\ell}-1}}\left(t_{i_{\ell}}\right) \delta_{n_{i_{\ell}} n_{I_{\ell}+1}} \cdots \delta_{n_{N-1} n_{N}}\right]+\mathcal{O}(\epsilon) \text {, }
\end{aligned}
$$

\footnotetext{
${ }^{1}$ Note that a sum of $N^{\ell}$ terms of order $\epsilon^{\ell}$ is of order $\epsilon^{0}=1$.
} 
where $\ell=0,1, \cdots, N$ and $t_{k}=k \epsilon / N=0, \epsilon, \cdots, \tau$. In particular,

$$
\begin{aligned}
K_{n_{N} n_{0}}^{(0)}(\tau) & =\Gamma_{n_{N}}(\tau) \delta_{n_{N} n_{0}}+\mathcal{O}(\epsilon) \\
K_{n_{N} n_{0}}^{(1)}(\tau) & =-\frac{i}{\hbar} \Gamma_{n_{N}}(\tau) \sum_{t_{i}=t_{j}}^{t_{k}} \epsilon H_{n_{N} n_{0}}^{\prime}\left(t_{i}\right)+\mathcal{O}(\epsilon) \\
& =-\frac{i}{\hbar} \sum_{m}\left[K_{n_{N} m}^{(0)}(\tau) \sum_{t_{i}=0}^{\tau} \epsilon H_{m n_{0}}^{\prime}\left(t_{i}\right)\right]+\mathcal{O}(\epsilon)
\end{aligned}
$$

where

$$
\begin{aligned}
\Gamma_{n}\left(t_{k}\right) & :=e^{\frac{i}{\hbar} \sum_{t_{j}=0}^{t_{k}}\left[-E_{n}\left(t_{j}\right)+i \hbar A_{n n}\left(t_{j}\right)\right] \epsilon}, \\
H_{m n}^{\prime}\left(t_{k}\right) & :=i \hbar \Gamma_{m}^{*}\left(t_{k}\right) \Delta_{m n}\left(t_{k}\right) \Gamma_{n}\left(t_{k}\right),
\end{aligned}
$$

and $\Gamma_{m}^{*}\left(t_{k}\right)=1 / \Gamma_{m}\left(t_{k}\right)$ is the complex conjugate of $\Gamma_{m}\left(t_{k}\right)$.

Furthermore, for every $\ell>1$, one can express $K_{m n}^{(\ell)}(\tau)$ in terms of $K_{m n}^{(0)}(\tau)$ and $H_{m n}^{\prime}\left(t_{i}\right)$, namely

$$
\begin{aligned}
K_{m m_{\ell}}^{(\ell)}(\tau) & =\left(\frac{-i}{\hbar}\right)^{\ell} \sum_{t_{i_{1}}<\cdots<t_{i_{\ell}}=0}^{\tau} \epsilon^{\ell} \sum_{m_{0} \cdots m_{\ell-1}} K_{m m_{0}}^{(0)}(\tau)\left[\prod_{a=1}^{\ell} H_{m_{a-1} m_{a}}^{\prime}\left(t_{i_{a}}\right)\right]+\mathcal{O}(\epsilon) \\
& =\frac{1}{\ell !}\left(\frac{-i}{\hbar}\right)^{\ell} \sum_{m_{0} \cdots m_{\ell-1}} K_{m m_{0}}^{(0)}(\tau) \sum_{t_{i_{1}} \cdots t_{i_{\ell}}=0}^{\tau} \epsilon^{\ell} \mathcal{T}\left[\prod_{a=1}^{\ell} H_{m_{a-1} m_{a}}^{\prime}\left(t_{i_{a}}\right)\right]+\mathcal{O}(\epsilon)
\end{aligned}
$$

Therefore if one defines:

$$
\begin{aligned}
U^{(\ell)}(\tau) & :=\lim _{N \rightarrow \infty} \sum_{m n} K_{m n}^{(\ell)}(\tau)|m ; \tau\rangle\langle n ; 0|, \\
H^{\prime}(t) & :=\lim _{N \rightarrow \infty} \sum_{m n} H_{m n}^{\prime}(t)|m ; 0\rangle\langle n ; 0|, \\
& =i \hbar \sum_{m n} e^{-i\left[\alpha_{m}(t)-\alpha_{n}(t)\right]} \Delta_{m n}(t)|m ; 0\rangle\langle n ; 0|, \\
& =-i \hbar \sum_{m \neq n} e^{-i\left[\alpha_{m}(t)-\alpha_{n}(t)\right]} A_{m n}(t)|m ; 0\rangle\langle n ; 0|,
\end{aligned}
$$

then

$$
U(\tau)=\sum_{\ell=0}^{\infty} U^{(\ell)}(\tau)=U^{(0)}(\tau)\left[\mathcal{T} e^{-\frac{i}{\hbar} \int_{0}^{\tau} d t H^{\prime}(t)}\right]
$$


where by taking $N \rightarrow \infty$ the sums of the form $\sum_{t_{i}=t}^{t^{\prime}} \epsilon f\left(t_{i}\right)$ have been promoted to the integrals $\int_{t}^{t^{\prime}} d t f(t)$. In particular one has:

$$
\begin{aligned}
U^{(0)}(\tau) & =\sum_{n} e^{i \alpha_{n}(\tau)}|n ; \tau\rangle\langle n ; 0| \\
U^{(1)}(\tau) & =U^{(0)}(\tau)\left[\frac{-i}{\hbar} \int_{0}^{\tau} d t H^{\prime}(t)\right] .
\end{aligned}
$$

By construction $U^{(0)}(\tau)$ yields $U(\tau)$ in the adiabatic approximation (5). Therefore, adiabatic approximation is valid only if one can neglect $\int_{o}^{\tau} d t H^{\prime}(t)$ in (27). It is not difficult to observe that this condition is fulfilled if the adiabaticity parameter $\nu$ as defined by Eq. (12) is negligible. In fact, for every $t_{1}$ and $t_{2}$ satisfying $0 \leq t_{1} \leq t_{2} \leq \tau$, one has:

$$
\left|\left\langle m ; 0\left|\int_{t_{1}}^{t_{2}} d t H^{\prime}(t)\right| n ; 0\right\rangle\right| \leq \int_{t_{1}}^{t_{2}} d t\left|\left\langle m ; 0\left|H^{\prime}(t)\right| n ; 0\right\rangle\right| \leq \hbar \int_{t_{1}}^{t_{2}} d t\left|A_{m n}(t)\right| \leq \Delta \tau \Delta E \nu
$$

where $\Delta \tau:=\tau_{2}-\tau_{1}$ with $\left[\tau_{1}, \tau_{2}\right] \subseteq[0, \tau]$ is the time interval over which $\omega_{c}$ is non-vanishing, i.e., $\tau_{1}$ and $\tau_{2}$ and therefore $\Delta \tau$ are defined by the condition:

$$
\omega_{c}\left(\tau_{1}^{\prime}, \tau_{2}^{\prime}\right)=0 \quad \text { if and only if } \quad \tau_{2}^{\prime}<\tau_{1} \text { or } \tau_{1}^{\prime}>\tau_{2}
$$

Note that usually $\Delta \tau$ is a finite time interval whereas $\tau$ may be infinite (arbitrarily large). A case for which $\Delta \tau=\tau$ is when the Hamiltonian depends periodically on time. In this case however, the physically interesting features of the evolution is given by $\Delta \tau=\tau \leq T$, where $T$ is the period of the Hamiltonian. Particularly interesting is the case $\tau=T$.

In general, $\Delta \tau$ may become arbitrarily large in which case (30) is a trivial statement. However, even in this case $\nu$ plays a most important role. In fact, one can argue that in general the following relation holds:

$$
U(\tau)=\sum_{\ell=0}^{N-1} U^{(\ell)}(\tau)+\mathcal{O}\left(\nu^{N}\right) .
$$

In particular the adiabatic approximation is valid if and only if $\nu \ll 1$. In order to establish Eq. (32), it is sufficient' to show that $U^{(1)}(\tau)$ and therefore $\int d t H^{\prime}(t)$ is at least of order $\nu$. This is however self-evident since one knows that if $\nu=0$, then $U(\tau)=U^{(0)}(\tau)$. Consequently, in a $\nu$-expansion of $U(\tau), U^{(1)}(\tau)$ is necessarily of order $\nu$ or higher.

\footnotetext{
${ }^{2}$ This is because $U^{(\ell)}(\tau)$ involves $\ell$ copies of $H^{\prime}(\tau)$ in its integrand.
} 
An alternative way of expressing Eq. (32) is to define $\tilde{U}^{(\ell)}(\tau):=U^{(\ell)}(\tau) / \nu$ and $\tilde{H}^{\prime}(\tau):=$ $H^{\prime}(\tau) / \nu$ and write $U(\tau)$ in the form:

$$
U(\tau)=\sum_{\ell=0}^{\infty} \tilde{U}^{(\ell)}(\tau) \nu^{\ell}=U^{(0)}(\tau)\left[\mathcal{T} e^{-\frac{i \nu}{\hbar} \int_{0}^{\tau} d t \tilde{H}^{\prime}(t)}\right] .
$$

This equation must not however be viewed as a true power series expansion of $U(\tau)$ in $\nu$, for $\tilde{U}^{(\ell)}(\tau)$ may in general depend on $\nu$.

A simple consequence of Eq. (33) is the fact that the essential ingredient which determines $U(\tau)$ beyond the adiabatic approximation is not $\tau$, but $\left(\tau_{1}, \tau_{2}\right)$ of (31) and $\nu$. For physically realistic non-periodic Hamiltonians, the latter quantities take finite values whereas the duration $\tau$ of the evolution of the system may be arbitrarily large. For a quantum system with a periodic Hamiltonian $H(t)=H(t+T)$, the physically interesting case is when $\tau_{2}=\tau \leq T$. In this case, $\nu$ may or may not be determined by $T$. This is because, in general the parameters $R_{C}^{\mu}(t)$ of the Hamiltonian have different periods. These are necessarily of the form $T_{\mu}=T / z_{\mu}$, respectively, where $z_{\mu}$ is a positive integer. Clearly $\omega_{c}(0, \tau)$ and consequently $\nu$ will depend on the largest value of $z_{\mu}$. Therefore, in general the statement that "the adiabatic approximation is valid if the period of the Hamiltonian is large," is false. The only case where $\nu$ depends on $T$ and the preceding statement is valid, is the case where the energy eigenstates are time-dependent and either there is effectively one changing parameter or $T_{\mu}=T$ for all $\mu$. Typical examples of these two cases are a spin in a precessing magnetic field [2] and a spin in a precessing and nutating magnetic field with equal precession and nutation periods, respectively.

Another useful observation is that in the eigenbasis $\{|n ; 0\rangle\}$ of $H(0), H^{\prime}(t)$ has no diagonal matrix elements, i.e.,

$$
\left\langle m ; 0\left|H^{\prime}(t)\right| n ; 0\right\rangle=\left\{\begin{array}{cc}
0 & \text { for } \quad m=n \\
i \hbar \frac{\left\langle m ; t\left|\left[\frac{d}{d t} H(t)\right]\right| n ; t\right\rangle}{E_{m}(t)-E_{n}(t)} e^{-i\left[\alpha_{m}(t)-\alpha_{n}(t)\right]} & \text { for } m \neq n,
\end{array}\right.
$$

where use is made of Eqs. (26) and (11). This equation also implies that the matrix elements $\left\langle m ; \tau\left|U^{(\ell)}(\tau)\right| n ; 0\right\rangle$ are directly related to $\left(\frac{1}{|n-m|}\right)^{\ell}$, i.e., the main contribution to $U^{(\ell)}(\tau)$ comes from the nearest energy levels. 


\section{Relation between $H^{\prime}(t)$ and $H(t)$}

Consider Eq. (26). Since the $m=n$ term is missing in the sum in (26), one can express $H^{\prime}(t)$ in the form:

$$
H^{\prime}(t)=-i \hbar \sum_{m \neq n}\left[\langle m ; t| e^{-i \alpha_{m}(t)}\right] \frac{d}{d t}\left[e^{i \alpha_{n}(t)}|n ; t\rangle\right]|m ; 0\rangle\langle n ; 0| .
$$

This equation together with the definition of $U^{(0)}(t)$ leads to

$$
H^{\prime}(t)=U^{(0) \dagger}(t) H(t) U^{(0)}(t)-i \hbar U^{(0) \dagger}(t) \frac{d}{d t} U^{(0)}(t) .
$$

The resemblance of this equation to the gauge transformations of the gauge potential in nonAbelian gauge theories (connections on principal fiber bundles) is remarkable, [⿴囗. $]$. Moreover, a simple calculation shows that

$$
\begin{aligned}
U^{(0) \dagger}(t) H(t) U^{(0)}(t) & =\sum_{n} E_{n}(t)|n ; 0\rangle\langle n ; 0| \\
H^{\prime}(t) & =-i \hbar \sum_{n \neq m}\left\langle m ; 0\left|U^{(0) \dagger}(t) \frac{d}{d t} U^{(0)}(t)\right| n ; 0\right\rangle|m ; 0\rangle\langle n ; 0|
\end{aligned}
$$

Therefore, the role of the first term on the right hand side of (36) is to cancel the diagonal matrix elements of the second term.

Eqs. (36) and (38) signify the importance of the adiabatic approximation in the determination of the non-adiabatic corrections $U^{(\ell)}(\tau)(\ell>0)$ to $U^{(0)}(\tau)$ and consequently the exact evolution operator $U(\tau)$.

In order to fully appreciate the meaning of Eq. (36), one should recall the effect of a general time-dependent unitary transformation of the Hilbert space $\mathcal{H}$. Let $\mathcal{U}(t): \mathcal{H} \rightarrow \mathcal{H}$ be such a transformation and suppose that the transformed state vectors

$$
|\breve{\psi}(t)\rangle:=\mathcal{U}(t)|\psi(t)\rangle
$$

satisfy the following Schrödinger equation:

$$
i \hbar \frac{d}{d t}|\check{\psi}(t)\rangle=\check{H}(t)|\check{\psi}(t)\rangle
$$


Then requiring $|\psi(t)\rangle$ to satisfy the Schrödinger equation defined by the original Hamiltonian $H(t)$, one finds:

$$
\check{H}(t)=\mathcal{U}(t) H(t) \mathcal{U}^{\dagger}(t)-i \hbar \mathcal{U}(t) \frac{d}{d t} \mathcal{U}^{\dagger}(t)
$$

Therefore Eq. (36) is a particular example of Eq. (41) with $\mathcal{U}(t)=U^{(0) \dagger}(t)$ and $\check{H}(t)=H^{\prime}(t)$. In other words, the non-adiabatic effects are given by a transformed Hamiltonian $H^{\prime}(t)$, where the transformation is performed by the inverse of the adiabatically approximate evolution operator, i.e., $U^{(0) \dagger}(t)$.

Transformation (39) may be used to yield an exact solution of the Schrödinger equation, if one can find a $\mathcal{U}(t)$ that makes the transformed Schrödinger equation (40) easily solvable. The Rabi-Ramsey-Schwinger method [5] is a special case of this approach where one uses the symmetry of the problem to find a unitary transformation $\mathcal{U}(t)$ which renders $\check{H}$ timeindependent. ${ }^{3}$ This is in general possible for cranked Hamiltonians with a fixed cranking direction [6, 7].

\section{Time-Dependent Unitary Transformations and the Geometric Phase}

There is another significant application of time-dependent unitary transformations of the Hilbert space when the Hamiltonian is $T$-periodic. In this case, one knows from the Floquet theory [8] that the evolution operator is given by $U(\tau)=Z(\tau) \exp [-i \tau \tilde{H} / \hbar]$, where $Z(\tau)$ is a $T$-periodic unitary operator with $Z(T)=Z(0)=1$, and $\tilde{H}$ is a time-independent Hermitian operator. Then by definition the cyclic states $\left.\left.\right|_{\mathcal{N}} ; T\right\rangle\left\langle_{\mathcal{N}} ; T\right|$ at $\tau=T$ are given by the eigenstates of $\tilde{H}$ and the associated total phase angles are $\beta_{\mathcal{N}}(T)=-T\left\langle_{\mathcal{N}} ; T|\tilde{H}|_{\mathcal{N}} ; T\right\rangle / \hbar$. Next consider choosing $\mathcal{U}(t)$ of Eq. (39) to be $Z^{\dagger}(t)$ and $\left.|\psi(0)\rangle=|\check{\psi}(0)\rangle=\left.\right|_{\mathcal{N}} ; T\right\rangle$. Then by virtue of Eq. (41), one has:

$$
\begin{aligned}
\beta_{\mathcal{N}}(T) & =-\frac{T}{\hbar}\langle\psi(0)|\tilde{H}| \psi(0)\rangle=T\left[-\frac{1}{\hbar}\langle\psi(t)|H(t)| \psi(t)\rangle+i\left\langle\psi(0)\left|Z^{\dagger}(t) \frac{d}{d t} Z(t)\right| \psi(0)\right\rangle\right], \\
& =\Delta_{\mathcal{N}}(T)+\Gamma_{\mathcal{N}}(T),
\end{aligned}
$$

\footnotetext{
${ }^{3}$ For a recent review of the application of this method to a spin system in a precessing magnetic field, see Ref. [2].
} 


$$
\begin{aligned}
\Delta_{\mathcal{N}}(\tau) & :=-\frac{1}{\hbar} \int_{0}^{\tau} d t\langle\psi(t)|H(t)| \psi(t)\rangle \\
\Gamma_{\mathcal{N}}(\tau) & :=i \int_{0}^{\tau} d t\left\langle\phi_{\mathcal{N}}(t)\left|\frac{d}{d t}\right| \phi_{\mathcal{N}}(t)\right\rangle
\end{aligned}
$$

where $|\phi(t)\rangle:=Z(t)|\psi(0)\rangle$. The phase angles $\Delta_{\mathcal{N}}(\tau)$ and $\Gamma_{\mathcal{N}}(\tau)$ were first introduced by Aharonov and Anandan [9], and called the dynamical and geometrical phase angles. They generalize their adiabatic counterparts previously discovered by Berry [1]. Clearly, for $\nu \ll 1$, one has: $|\mathcal{N} ; T\rangle \approx|n ; 0\rangle, \Delta_{\mathcal{N}}(T) \approx \delta_{n}(T)$, and $\Gamma_{\mathcal{N}}(T) \approx \gamma_{n}(T)$.

Next consider the general case. If $\mathcal{U}(t)$ is also assumed to leave the original energy eigenstates invariant, i.e., $\check{H}(t)$ has the same eigenstates as $H(t)$, then one can show that $\mathcal{U}(t)$ and $\check{H}(t)$ must be of the form:

$$
\begin{aligned}
& \mathcal{U}(t)=e^{i f(t)} \mathcal{U}_{0}, \\
& H(t) \rightarrow \check{H}(t)=H(t)-\hbar \frac{d f(t)}{d t} 1,
\end{aligned}
$$

where $f=f(t)$ is a real-valued function, $\mathcal{U}_{0}$ is a constant unitary transformation, and 1 is the identity operator on $\mathcal{H}$. Note that transformation (39) with $\mathcal{U}(t)$ given by (45) leaves all the observables invariant. It shifts the energy eigenvalues:

$$
E_{n}(t) \rightarrow E_{n}^{\prime}(t)=E_{n}(t)-\hbar \frac{d f(t)}{d t},
$$

but does not affect the observable transition energies $E_{n}(t)-E_{m}(t)$. Therefore, one can identify a physical system with the equivalence class of all the quantum systems which are related with transformations of the form (39,45).

A simple consequence of this observation is that unlike the dynamical phase angle (43), the geometric phase angle (44) is a physical quantity [2]. This statement however requires a clarification. One can easily see that (46) changes the dynamical phase angle $\Delta_{\mathcal{N}}(\tau)$. It does nevertheless leave the difference of two dynamical phase angles and consequently two total phase angles invariant. Therefore, the dynamical phase angle also carries physically significant information about the evolving system. In fact, a close look at the experimental results on the detection of the geometric phase [10] clearly shows that the measurable

\footnotetext{
${ }^{4}$ Note that in general the Hamiltonian needs not be periodic and the Floquet theory does not apply. However, even in this case one can define $\left|\phi_{\mathcal{N}}(t)\right\rangle$ and Eqs. (42)-(44) are valid. See [9, 2] for more detail.
} 
quantities are related to the differences of total phase angles. This is usually overshadowed by the fact that in the best studied system, namely the two-level spin $(j=1 / 2)$ system in a precessing magnetic field [1], the conventional choice of the Hamiltonian (48) leads to two cyclic states whose total phase angles differ by a minus sign. Therefore their difference is twice one of them and it appears that the experiments detect a total phase. In reality however, the experiments always detect the differences between total phase angles, a quantity which is invariant under (46). This can be easily seen if one uses a complete orthonormal set of cyclic states $\left.\left\{\left.\right|_{\mathcal{N}} ; \tau\right\rangle\right\}$ to compute the expectation values:

$$
\begin{aligned}
\langle X(\tau)\rangle & :=\langle\psi(\tau)|X(0)| \psi(\tau)\rangle=\left\langle\psi(0)\left|U^{\dagger}(\tau) X(0) U(\tau)\right| \psi(0)\right\rangle, \\
& =\sum_{\mathcal{N}, \mathcal{N}^{\prime}}\left\langle\left.\psi(0)\right|_{\mathcal{N}} ; \tau\right\rangle\left\langle\mathcal{N}_{\mathcal{N}} ; \tau\left|U^{\dagger}(\tau) X(0) U(\tau)\right|_{\mathcal{N}^{\prime}}, \tau\right\rangle\left\langle\mathcal{N}^{\prime} ; \tau \mid \psi(0)\right\rangle \\
& =\sum_{\mathcal{N}^{\prime}, \mathcal{N}^{\prime}} e^{-i\left[\beta_{\mathcal{N}}(\tau)-\beta_{\mathcal{N}^{\prime}}(\tau)\right]}\left\langle\left.\psi(0)\right|_{\mathcal{N}} ; \tau\right\rangle\left\langle{ }_{\mathcal{N}} ; \tau|X(0)|_{\mathcal{N}^{\prime}}, \tau\right\rangle\left\langle\mathcal{N}^{\prime} ; \tau \mid \psi(0)\right\rangle
\end{aligned}
$$

where $X(0)$ is an observable and in the last equality used is made of Eq. (11).

Incidentally, it is not too difficult to see that by definition, the adiabaticity parameter $\nu$ (12), the operator $H^{\prime}(t)$ of Eq. (26), and consequently all the non-adiabatic corrections $U^{(\ell)}(\tau)$ (with $\ell \geq 1$ ) to $U^{(0)}(\tau)$ are invariant under the transformation (45). Therefore all of these quantities signify physically measurable effects.

\section{Application to a Magnetic Dipole in a Magnetic Field}

In this section, the utility of the expansion (27) is demonstrated for the quantum system consisting of a magnetic dipole moment subject to a magnetic field with changing direction.

The Hamiltonianf of this system is given by:

$$
H(\theta, \varphi)=b \vec{R}(\theta, \varphi) \cdot \vec{J}=b\left(\sin \theta \cos \varphi J^{1}+\sin \theta \sin \varphi J^{2}+\cos \theta J^{3}\right),
$$

where $b$ is the Larmor frequency, $\theta$ and $\varphi$ are the azimuthal and polar angles in spherical coordinates, respectively, and $\vec{J}$ is the angular momentum operator with components $J^{\mu}$, $\mu=1,2,3$. Clearly the parameter manifold is the two-dimensional sphere $S^{2}$ and the timedependence of the Hamiltonian is described by a curve $C:[0, \tau] \rightarrow S^{2}$. Without loss of

\footnotetext{
${ }^{5}$ As discussed in the previous section, the choice of a Hamiltonian is not unique, i.e., one can add a multiple of the identity operator to Eq. (3.1) without having any physical consequences.
} 
generality one can choose a coordinate system in which $C$ does not pass through the south pole. This allows one to work with a single coordinate patch of $S^{2}$ which excludes the south pole.

In this patch one has [2]:

$$
\begin{aligned}
E_{n}(\theta, \varphi) & =E_{n}(0,0)=b \hbar n, \quad \text { with } n=0, \pm \frac{1}{2}, \pm 1, \pm \frac{3}{2}, \cdots, \\
|n ;(\theta, \varphi)\rangle & =e^{-\frac{i \varphi}{\hbar} J^{3}} e^{-\frac{i \theta}{\hbar} J^{2}} e^{\frac{i \varphi}{\hbar} J^{3}}|n ;(0,0)\rangle, \quad \theta \in[0, \pi), \varphi \in[0,2 \pi) .
\end{aligned}
$$

By definition $|n ;(0,0)\rangle$ are the eigenvectors of $H(\theta=0, \varphi=0)=b J^{3}$, i.e.,

$$
J^{3}|n ;(0,0)\rangle=\hbar n|n ;(0,0)\rangle .
$$

In order to compute the operators $U^{(0)}(\tau)$ and $H^{\prime}(\tau)$, one first calculates

$$
\begin{aligned}
A_{m n}(t) & =A_{\theta}^{(m n)} \dot{\theta}(t)+A_{\varphi}^{(m n)} \dot{\varphi}(t) \\
A_{\theta}^{(m n)} & :=\left\langle m ;(\theta, \varphi)\left|\frac{\partial}{\partial \theta}\right| n ;(\theta, \varphi)\right\rangle, \\
& =\frac{i}{\hbar}\left[\sin \varphi\left\langle J_{m n}^{1}\right\rangle_{0}-\cos \varphi\left\langle J_{m n}^{2}\right\rangle_{0}\right], \\
A_{\varphi}^{(m n)} & :=\left\langle m ;(\theta, \varphi)\left|\frac{\partial}{\partial \varphi}\right| n ;(\theta, \varphi)\right\rangle, \\
& =i\left[m(1-\cos \theta) \delta_{m n}+\frac{1}{\hbar} \sin \theta\left(\cos \varphi\left\langle J_{m n}^{1}\right\rangle_{0}+\sin \varphi\left\langle J_{m n}^{2}\right\rangle_{0}\right)\right],
\end{aligned}
$$

where $\left\langle J_{m n}^{\mu}\right\rangle_{0}:=\left\langle m ;(0,0)\left|J^{\mu}\right| n ;(0,0)\right\rangle,(\theta(t), \varphi(t))=C(t)$ and dot stands for $d / d t$. In the derivation of Eqs. (52) and (53) use is made of the following identities:

$$
\begin{aligned}
e^{-\frac{i \varphi}{\hbar} J^{3}} J^{2} e^{\frac{i \varphi}{\hbar} J^{3}} & =-\sin \varphi J^{1}+\cos \varphi J^{2}, \\
e^{\frac{i \theta}{\hbar} J^{2}} J^{3} e^{-\frac{i \theta}{\hbar} J^{2}} & =\cos \theta J^{3}-\sin \theta J^{1}, \\
e^{-\frac{i \varphi}{\hbar} J^{3}} J^{1} e^{\frac{i \varphi}{\hbar} J^{3}} & =\cos \varphi J^{1}+\sin \varphi J^{2} .
\end{aligned}
$$

Next step is the calculation of the phase angles $\alpha_{n}(t)$ of Eq. (7). These are given by:

$$
\alpha_{n}(t)=\delta_{n}(t)+\gamma_{n}(t), \quad \delta_{n}(t)=-b t n, \quad \gamma_{n}(t)=-n \gamma(t),
$$

where

$$
\gamma(t):=\int_{0}^{t} d t^{\prime}(1-\cos \theta) \dot{\varphi}=\int_{0}^{\varphi(t)}(1-\cos \theta) d \varphi .
$$


In (55), $\varphi(0)$ is set to zero and in the second integral $\varphi$ is used to parameterize the curve $C$, i.e., $\theta=\theta(\varphi)$.

Eqs. (51-53), (54), and (55) together with Eqs. (28) and (26) yield:

$$
\begin{aligned}
U^{(0)}(\tau) & =\sum_{n} e^{-i[b \tau+\gamma(\tau)] n}|n ; \tau\rangle\langle n ; 0| \\
H^{\prime}(t) & =\sum_{m \neq n}\left[H_{1}^{\prime m n}(t)\left\langle J_{m n}^{1}\right\rangle_{0}+H_{2}^{\prime m n}(t)\left\langle J_{m n}^{2}\right\rangle_{0}\right]|m ; 0\rangle\langle n ; 0|,
\end{aligned}
$$

where

$$
\begin{aligned}
& H_{1}^{\prime m n}(t):=e^{i[b t+\gamma](m-n)}(\sin \varphi \dot{\theta}+\sin \theta \cos \varphi \dot{\varphi}) \\
& H_{2}^{\prime m n}(t):=e^{i[b t+\gamma](m-n)}(-\cos \varphi \dot{\theta}+\sin \theta \sin \varphi \dot{\varphi})
\end{aligned}
$$

In order to further ease the computation of $H^{\prime}(\tau)$, one can make the additional assumption that the energy eigenstates have definite total angular momentum, i.e.,

$$
|\vec{J}|^{2}|n,(\theta, \varphi)\rangle=j(j+1)|n,(\theta, \varphi)\rangle, \quad n=-j,-j+1, \cdots, j
$$

This assumption is too restrictive for the applications in molecular physics, where one encounters systems with cylinderical symmetry rather than spherical symmetry [2]. For a magnetic dipole in a classical environment, however, one can safely make this assumption. In this case, one can use the well-known relations [11]:

$$
J^{ \pm}:=J^{1} \pm i J^{2}, \quad J^{ \pm}|n ;(0,0)\rangle=\hbar C_{ \pm n}|n \pm 1 ;(0,0)\rangle, \quad C_{m}:=\sqrt{(j-m)(j-m+1)}
$$

to compute $\left\langle J_{m n}^{1}\right\rangle_{0}$ and $\left\langle J_{m n}^{2}\right\rangle_{0}$. This leads to

$$
\left\langle J_{m n}^{1}\right\rangle_{0}=\frac{\hbar}{2}\left(C_{n} \delta_{m n+1}+C_{-n} \delta_{m n-1}\right), \quad\left\langle J_{m n}^{2}\right\rangle_{0}=\frac{-i \hbar}{2}\left(C_{n} \delta_{m n+1}-C_{-n} \delta_{m n-1}\right) .
$$

Substituting Eqs. (62) and carrying out the necessary algebra one has:

$$
\begin{aligned}
A_{\theta}^{(m n)} & =\frac{1}{2}\left(e^{i \varphi} C_{m} \delta_{m n-1}-e^{-i \varphi} C_{n} \delta_{m-1 n}\right) \\
A_{\varphi}^{(m n)} & =i\left[m(1-\cos \theta) \delta_{m n}+\frac{1}{2} \sin \theta\left(e^{i \varphi} C_{m} \delta_{m n-1}+e^{-i \varphi} C_{n} \delta_{m-1 n}\right)\right] \\
H^{\prime}(t) & =\frac{\hbar}{2}\left[h^{\prime}(t) \sum_{n} C_{n}|n+1 ; 0\rangle\left\langle n ; 0\left|+h^{\prime *}(t) \sum_{n} C_{n}\right| n-1 ; 0\right\rangle\langle n ; 0|\right]
\end{aligned}
$$




$$
\begin{aligned}
= & \frac{\hbar}{2}\left[h^{\prime}(t) e^{-i \frac{\theta_{0}}{\hbar} J^{2}} \sum_{n} C_{n}|n+1 ;(0,0)\rangle\langle n ;(0,0)| e^{i \frac{\theta_{0}}{\hbar} J^{2}}+\right. \\
& \left.h^{\prime *}(t) e^{-i \frac{\theta_{0}}{\hbar} J^{2}} \sum_{n} C_{n}|n-1 ;(0,0)\rangle\langle n ;(0,0)| e^{i \frac{\theta_{0}}{\hbar} J^{2}}\right], \\
= & \frac{1}{2}\left[h^{\prime}(t) e^{-i \frac{\theta_{0}}{\hbar} J^{2}} J^{+} e^{i \frac{\theta_{0}}{\hbar} J^{2}}+h^{\prime *}(t) e^{-i \frac{\theta_{0}}{\hbar} J^{2}} J^{-} e^{i \frac{\theta_{0}}{\hbar} J^{2}}\right], \\
= & \operatorname{Re}\left[h^{\prime}(t)\right]\left(\cos \theta_{0} J^{1}-\sin \theta_{0} J^{3}\right)-\operatorname{Im}\left[h^{\prime}(t)\right] J^{2},
\end{aligned}
$$

where

$$
h^{\prime}(t):=e^{i[b t+\gamma-\varphi]}(i \dot{\theta}+\sin \theta \dot{\varphi})
$$

$\theta_{0}:=\theta(0)$, and "Re" and "Im" stand for the real part and ( $-i$ times) the imaginary part of the argument. Furthermore, in the derivation of (65) use is made of Eqs. (57), (50), (61), $\varphi(0)=0$, and the identities:

$$
e^{-i \frac{\theta_{0}}{\hbar} J^{2}} J^{ \pm} e^{i \frac{\theta_{0}}{\hbar} J^{2}}=e^{-i \frac{\theta_{0}}{\hbar} J^{2}} J^{1} e^{i \frac{\theta_{0}}{\hbar} J^{2}} \pm i J^{2}=\cos \theta_{0} J^{1}-\sin \theta_{0} J^{3} \pm i J^{2}
$$

Next consider the case in which the magnetic field performs a simultaneous precession and nutation, i.e.,

$$
C: \varphi=\omega t, \quad \cos \theta=\cos \theta_{0}+\eta \sin (l \omega t) .
$$

Here $\omega$ is the frequency of precession, and $|l| \omega$ and $\eta$ are the frequency and amplitude of the nutation, respectively. Clearly $0 \leq \eta<1 \pm \cos \theta_{0}$ and $l$ is an integer.

The computation of $\gamma$ is then straightforward:

$$
\gamma(t)=\left(1-\cos \theta_{0}\right) \omega t-\frac{\eta}{l}[1-\cos (l \omega t)] .
$$

Hence for $\tau=T:=2 \pi / \omega$, one recovers Berry's result:

$$
\gamma_{n}(T)=-2 \pi n\left(1-\cos \theta_{0}\right)=-n \Omega(C)
$$

where $\Omega(C)$ is the solid angle subtended by the curve $C$. A simple consequence of (70) is that the adiabatic approximation does not detect the effects of the nutation. In fact, one has:

$$
U^{(0)}(T)=\sum_{n} e^{-i\left[b T+2 \pi\left(1-\cos \theta_{0}\right)\right] n}|n ; 0\rangle\langle n ; 0|=e^{-i \frac{\theta_{0}}{\hbar} J^{2}} e^{-\frac{i}{\hbar}\left[b T+2 \pi\left(1-\cos \theta_{0}\right)\right] J^{3}} e^{i \frac{\theta_{0}}{\hbar} J^{2}} .
$$


The first non-adiabatic correction $U^{(1)}(T)$ to (71) is obtained by integrating $H^{\prime}(t)$ of Eq. (65). This involves the evaluation of

$$
I(T):=\int_{0}^{T} h^{\prime}(t) d t=\int_{0}^{T} d t e^{i[b t+\gamma-\varphi]}(i \dot{\theta}+\sin \theta \dot{\varphi}) .
$$

In order to give a closed expression for this integral and subsequently $U^{(1)}(T)$, one can expand the integrand in powers of $\eta$. This leads to a lengthy calculation which results in:

$$
\begin{aligned}
\operatorname{Re}[I(T)]= & \zeta\left\{\sin \theta_{0} \sin (2 \pi / \zeta)+\left[\left(\frac{1-\cos (2 \pi / \zeta)}{1-(l \zeta)^{2}}\right)\left(\frac{1}{\sin \theta_{0}}+\cot \theta_{0} \zeta-\sin \theta_{0} \zeta^{2}\right)\right] l \eta+\right. \\
& {\left.\left[\frac{2 \cos \theta_{0} \sin (2 \pi / \zeta) \zeta}{\sin ^{3} \theta_{0}\left[1-4(l \zeta)^{2}\right]}\right](l \eta)^{2}\right\}+\mathcal{O}\left(l \eta^{2}\right), } \\
\operatorname{Im}[I(T)]= & \zeta\left\{\sin \theta_{0}[1-\cos (2 \pi / \zeta)]+\left[\left(-\frac{1}{\sin \theta_{0}}-\cot \theta_{0} \zeta+\sin \theta_{0} \zeta^{2}\right)\left(\frac{\sin (2 \pi / \zeta}{1-(l \zeta)^{2}}\right)\right] l \eta+\right. \\
& {\left.\left[\frac{2 \cos \theta_{0}[1-\cos (2 \pi / \zeta)] \zeta}{\sin ^{3} \theta_{0}\left[1-4(l \zeta)^{2}\right]}\right](l \eta)^{2}\right\}+\mathcal{O}\left(l \eta^{2}\right), }
\end{aligned}
$$

where $\zeta:=\omega /\left(b-\omega \cos \theta_{0}\right)$. In terms of $\operatorname{Re}[I(T)]$ and $\operatorname{Im}[I(T)], U^{(1)}(T)$ is expressed in the form:

$$
\begin{aligned}
U^{(1)}(T) & =-\frac{i}{\hbar} U^{(0)}(T)\left\{\operatorname{Re}[I(T)]\left(\cos \theta_{0} J^{1}-\sin \theta_{0} J^{3}\right)-\operatorname{Im}[I(T)] J^{2}\right\}, \\
& =-\frac{i}{\hbar} e^{-i \frac{\theta_{0}}{\hbar} J^{2}} e^{-\frac{i}{\hbar}\left[b T+2 \pi\left(1-\cos \theta_{0}\right)\right] J^{3}}\left\{\operatorname{Re}[I(T)] J^{1}-\operatorname{Im}[I(T)] J^{2}\right\} e^{i \frac{\theta_{0}}{\hbar} J^{2}} .
\end{aligned}
$$

In particular, consider the special case, $\theta_{0}=\pi / 2$. Then $\zeta=\omega / b$ and Eqs. (71) and (73-75) reduce to:

$$
\begin{aligned}
U^{(0)}(T) & =e^{-i \frac{\pi}{2 \hbar} J^{2}} e^{-\frac{i}{\hbar}(b T+2 \pi) J^{3}} e^{i \frac{\pi}{2 \hbar} J^{2}}=e^{-i 2 \pi j} e^{-i \frac{\pi}{2 \hbar} J^{2}} e^{-\frac{i}{\hbar} b T J^{3}} e^{i \frac{\pi}{2 \hbar} J^{2}} \\
\operatorname{Re}[I(T)] & =\zeta\left\{\sin (2 \pi / \zeta)+\left[\left(\frac{1-\zeta^{2}}{1-(l \zeta)^{2}}\right)[1-\cos (2 \pi / \zeta)]\right] \operatorname{l\eta }\right\}+\mathcal{O}\left(l \eta^{2}\right) \\
\operatorname{Im}[I(T)] & =\zeta\left\{1-\cos (2 \pi / \zeta)+\left[\left(-\frac{1-\zeta^{2}}{1-(l \zeta)^{2}}\right) \sin (2 \pi / \zeta)\right] \operatorname{l\eta }\right\}+\mathcal{O}\left(l \eta^{2}\right) \\
U^{(1)}(T) & =-\frac{i}{\hbar} e^{-i 2 \pi j} e^{-i \frac{\pi}{2 \hbar} J^{2}} e^{-\frac{i}{\hbar} b T J^{3}}\left\{\operatorname{Re}[I(T)] J^{1}-\operatorname{Im}[I(T)] J^{2}\right\} e^{i \frac{\pi}{2 \hbar} J^{2}} .
\end{aligned}
$$

The adiabaticity parameter $\nu$ of Eq. (12) can also be easily calculated in this case:

$$
\nu=c \sqrt{1+(l \eta)^{2}} \zeta+\mathcal{O}\left(l^{2}\right)
$$


where $c:=C_{-j} / 2=\sqrt{j(j+1 / 2)}$. The appearance of $\zeta$ in this expression, is an indication of the fact that the leading order term in $U^{(1)}(T)$ is of order $\nu$. In fact, for the two extreme cases: $l \eta \ll 1$ and $l \eta \gg 1$ (with $l \eta^{2} \ll 1$ so that one can neglect $\mathcal{O}\left(l \eta^{2}\right)$ ) this is manifestly seen.

As indicated by Eqs. (76-79), the nutation of the direction of the magnetic field only contributes to the non-adiabatic part of the evolution operator.

\section{Summary and Conclusion}

In this article, I have tried to address some of the basic issues regarding the meaning of the adiabatic approximation and the implications of their resolution to the phenomenon of the geometric phases. A precise definition of an adiabaticity parameter, although being rather unimportant in practice, provides an objective criterion for the applicability of the adiabatic approximation. It also allows one to deal with some of the misconceptions concerning the subject. Specifically, it is shown that there is no relation between the length of the duration of the evolution and the validity of the adiabatic approximation. Furthermore for periodic Hamiltonians, the relation between the adiabaticity of the evolution and the period of the Hamiltonian is shown to be not a simple proportionality. For example for the system described by Eqs. (48) and (68), both the period $T$ and the nutation parameter $l \eta$ might be arbitrarily large, in which case the adiabatic approximation is not valid. An example of a periodic system with an arbitrary small period for which the adiabatic approximation is exact, is a system with constant energy eigenstates but time dependent energy eigenvalues. A typical example of such a system is a magnetic dipole in a magnetic field whose magnitude is rapidly changing but its direction is fixed. Clearly, in this case, the adiabaticity parameter $\nu$ vanishes identically and the adiabatic approximation is valid exactly.

A perturbative expansion of the evolution operator $U(\tau)$ is also proposed. This expansion generalizes the adiabatic approximation and computes the non-adiabatic corrections to the adiabatically approximate expression for $U(\tau)$. Furthermore, it separates the adiabatic and non-adiabatic parts of $U(\tau)$ and yields the non-adiabatic part as a time-ordered exponential defined by a Hermitian operator $H^{\prime}(t)$. In a basis which diagonalizes the initial Hamiltonian, $H^{\prime}(t)$ is off-diagonal. Moreover, the dominant contribution to this operator and consequently 
to the non-adiabatic part of the evolution operator comes from the nearest energy levels. Particularly interesting is to view $H^{\prime}(t)$ as a transformed Hamiltonian. This corresponds to a time-dependent unitary transformation of the state vectors which undoes the adiabatic part of the evolution operator.

The subject of the general time-dependent unitary transformations of the state vectors has also been reviewed and a simple application of such a transformation is used to yield a splitting of the total phase of a cyclic state into its dynamical and geometric parts for a periodically changing Hamiltonian. This is followed by a discussion of physically equivalent quantum systems and the conclusion that unlike the dynamical (total) phase of a cyclic state, the difference of the dynamical (total) phases of two cyclic states is a physical quantity.

Finally, the theoretical developments have been applied to the analysis of a quantum system consisting of a magnetic dipole subject to a magnetic field. In particular, an explicit formula for the operator $H^{\prime}(t)$ has been derived for the general case and the case of a precessing and nutating magnetic field has been studied in detail.

\section{Acknowledgements}

I would like to thank Bahman Darian and Rouzbeh Allahverdi for interesting discussions, and acknowledge the support of the Killam Foundation of Canada. 


\section{References}

[1] M. V. Berry, Proc. Roy. Soc. London A392, 45 (1984).

[2] A. Bohm, Quantum Mechanics: Foundations and Applications, third ed., chapters 22 and 23, Springer-Verlag, New York (1993). For more detail see Ref. [3].

[3] A. Bohm, A. Mostafazadeh, and J. Zwanziger, Quatum Geometric Phase, in preparation.

[4] M. Nakahara, Geometry, Topology and Physics, Adam Hilger (1990).

[5] I. I. Rabi, N. F. Ramsey, and J. Schwinger, Rev. Mod. Phys. 26, 167 (1954).

[6] S. J. Wang, Phys. Rev. A42, 5107 (1990).

[7] A. Mostafazadeh, J. Math. Phys. 37, 1218 (1996).

[8] D. J. Moore, J. Phys. A: Math. Gen. 23, L665 (1990). For more detail see Ref. [3].

[9] Y. Aharonov and J. Anandan, Phys. Rev. Lett. bf 58, 1593 (1987); J. Anandan and Y. Aharonov, Phys. Rev. D38, 1863 (1988).

[10] T. Bitter and D. Dubbers, Phys. Rev. Lett. 59, 251 (1987); D. Suter, K. T. Muller, and A. Pines, Phys. Rev. Lett. 60, 1218 (1988). For more detail see Ref. [3].

[11] J. J. Sakurai, Modern Quantum Mechanics, Addison-Wesley, New York (1985). 